\title{
ICT SOLUTIONS FOR LOCAL FLEXIBILITY MARKETS
}

\author{
Jin XIAOLONG \\ Technical University of Denmark \\ xiajin@elektro.dtu.dk \\ Simona-Vasilica OPREA \\ Bucharest University of Economic Studies \\ simona.oprea@csie.ase.ro \\ Adela BÂRA \\ Bucharest University of Economic Studies \\ bara.adela@ie.ase.ro \\ Vlad DIACONIȚA \\ Bucharest University of Economic Studies \\ diaconita.vlad@ie.ase.ro \\ Qiuwei WU \\ Technical University of Denmark \\ qw@elektro.dtu.dk
}

\begin{abstract}
Most of the Renewable Energy Sources (RES) and changing consumption patterns bring more volatility and fluctuations for retailers and distribution system operators. Such challenges are expected to increase in the coming years as the policies are constantly promoting RES. The Local Flexibility Market (LFM) is envisioned as an innovative solution to control the imbalances that usually appear between contractual agreements and short-term forecast. Consumers' flexibility to control their programmable appliances and prosumers' flexibility to control the Distributed Energy Resources (DER) including distributed generation, controllable loads, batteries, etc. and urban buildings have to be rewarded as they substantially contribute to the imbalance reduction. Hence, the LFM encourages investment in flexibilities such as controllable devices, smart plugs and storage facilities fostering the market competition. The communication system between consumers/prosumers and grid operators, and the connectivity of controllable appliances are essential to make such markets operational. In this context, ICT platforms offer secure trading opportunities to the local flexibility market players such as consumers, prosumers, retailers and DSO. In this paper, we will focus on the ICT architecture for trading the flexibilities.
\end{abstract}

Keywords: local flexibility market, prosumers, storage providers, ICT solution, Blockchain JEL classification: P18, D44, D47, L86

DOI: $10.24818 / \mathrm{ie} 2020.03 .05$

\section{Introduction}

Grid congestions are identified usually after market simulation is performed as it does not take into account the grid limits. They are identified by grid simulations that consider both the market results (the merit order) and grid limits when thermal limits of the conductors are exceeded. Thus, in real operation grid congestions should be avoided by building additional grid capacities on long-term or by redispathing the generators to ensure that transmission and distribution of the electricity is under the standard parameters and supply the residential and industrial consumption areas in proper conditions. Usually, cheap RES generate large volume 
of electricity in weak grid areas that connect the final consumers. Also, RES has a highly volatile operation that requires power system reserve capacities to balance the fluctuating output of RES. Also, the forecast errors lead to significant and costly market imbalances that should be also avoided. Such issues could be analysed considering the flexibility services that could be locally traded and rewarded.

Since there are different entities such as: Transmission System Operators (TSOs) that manage the power system balancing, frequency control and congestion management; Distribution System Operators (DSOs) also handle the congestion management and voltage regulation [1], [2] and retailer the imbalance minimization, various flexibility products and markets can be envisioned. For TSOs, the ancillary services market and balancing market cope with TSOs concerns that are more at the power system level, while DSOs have to encounter local voltage and congestion issues that could be solved by implementing the concept of LFM. Also, retailers have to cope with load fluctuations and volatility of RES, seeking to reduce the imbalances between the acquired electricity and forecast that generate additional costs [3]. The acquisition of flexibility services via bilateral contracts [4] or bidding systems [5], [6] are envisioned for trading the flexibilities at the retailers or DSOs level. Flexibility services are characterized by several particularities, such as capacity, ramp rate, direction (up or down), starting time, trigger, duration, and location (grid node) as in Fig. 1.

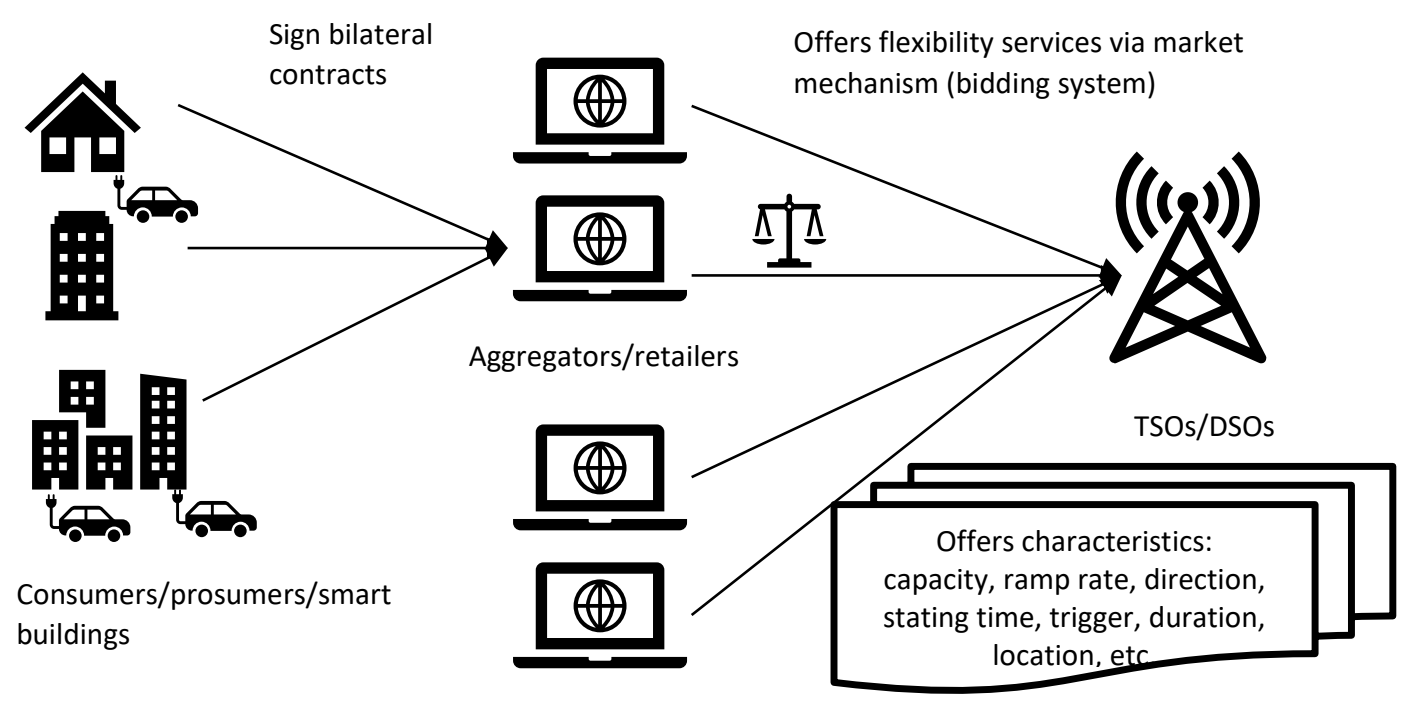

Figure 1. Market players of an LFM and offers characteristics.

The paper is structured in four sections: introduction, local flexibility markets concept, ICT solutions for LFM, and finally, conclusions.

\section{Concepts of local flexibility market 2.1 Concepts}

Local electricity flexibility markets are described in [2], [7] considering three scenarios regarding flexibility requirements intervals and direction showing the applicability and advantages of the market mechanism. Three variants of market design for trading the local flexibility that handles grid and balancing issues are identified in [8]: a) trading flexibility together with the wholesale market; b) distinct flexibility market; c) the power system reserve capacity market.

A framework for LFM with two stages, planning and real-time is proposed in [9] and [10] enabling DSO to acquire the cheapest flexibility for managing grid issues. 
In LFM, [11] underlines the specific contractual aspects between DER and DSO, and processes, assessing their efficacity in the market. However, the implementation of LFM faces several issues, such as: legislative gaps, obstacles and barriers identified in [12].

Trading platforms for prosumers to integrate them into intra-day markets and residential storage facilities to enhance the local flexibility are envisiond in [13]. An interaction platform for flexibility service providers and potential beneficiaries is also proposed in [14].

A local flexibility market (LFM) can be defined as a flexibility products or services trading platform in geographically limited areas [7]. In this context, an LFM includes products or services to be traded in the LFM, market operator, market participants, and market clearing mechanism [1]. Figure shows an LFM where a DSO, a balance responsible party (BRP) and several aggregators trade flexibility locally under the supervision of an LFM operator. Market participants in the LFM include a DSO, a BRP, aggregators, and an LFM operator. As can be observed from Figure, the DSO and BRP buy flexibility for different purposes: congestion management and loss reduction for the DSO; optimizing the portfolio and reducing imbalance volumes for the BRP. The aggregators sell the flexibility for profits by modifying their electricity usage upon request and earn profits based on flexibility transactions.

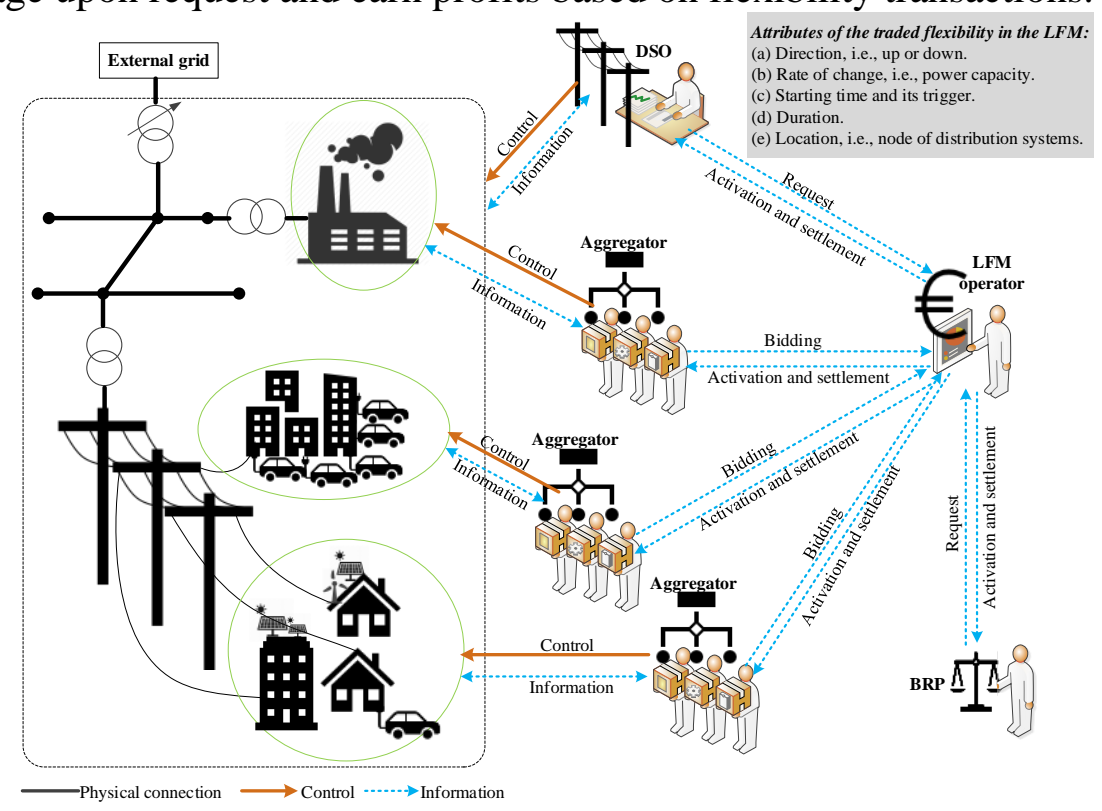

Figure 2. Schematic overview of an LFM (adopted from [1]).

\subsection{Key elements and technologies}

Ref. [1] proposed a four-layer structure for LFMs to show the potential key technologies used in the LFMs. It includes the power grid layer, the ICT layer, the control layer, and the market layer. Each layer of the four-layer structure is introduced as follows:

(1) The power grid layer is composed of all the physical components of the electric distribution system. These components compose the electric distribution system where local flexibility trading can be conducted.

(2) The ICT layer is composed of communication devices, protocols, applications, and information flow to support local flexibility trading [15]. The ICT layer provides ICT infrastructure for local flexibility trading, which is the main focus of this paper.

(3) The control layer is mainly composed of the control functions for the distribution system. The control layer is mainly responsible for the management of the flexibility orders decided in the market layer. 
www.conferenceie.ase.ro

(4) The market layer determines how flexibility is traded locally among the participants in the LFM. The market layer is responsible for the management of flexibility transactions in the LFM.

Jin et al. [1] presented a detailed review of concepts, formulations, and clearing methods of LFMs. For more details regarding the LFM, one can refer to [1]. This paper will mainly focus on the ICT designs for LFMs.

\section{ICT solutions}

For LFM, there are different information flows within the different actors (LFM operator, BRP, DSO, Aggregators). To support these information flows, the proposed architecture (Fig. 3) components are:

- A streaming solution that supports message queues and publish-subscribe messaging patterns;

- A ledgering solution such as Blockchain for the P2P trading;

- A data storage solution structured on different layers:

o Database tier (relational or NoSQL) - for current operational data that are not appropriate to be stored in a

- Datawarehouse tier - for reporting and historical analyses

$\circ$ Archival tier - for long time storage of data

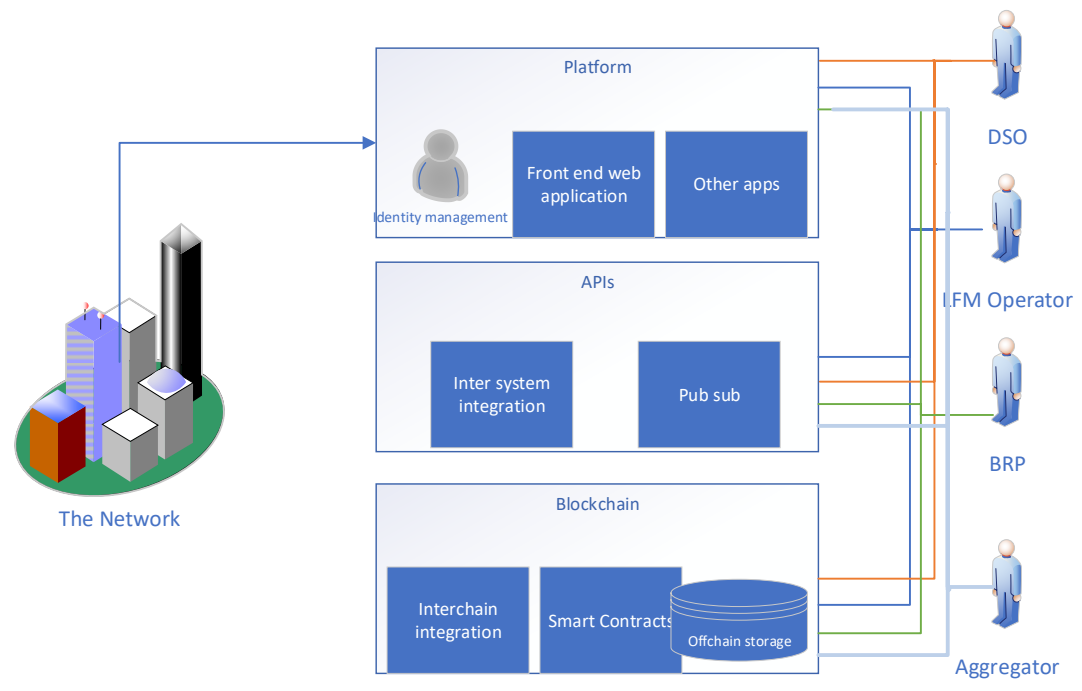

Figure 3. The Blockchain centered architecture

An excellent choice for P2P trading backend is Blockchain, which is a distributed and decentralized ledger system that can offer integrity and transparency. It can be implemented from scratch or from using Blockchain-as-a-Service solutions from the major Cloud vendors (AWS, Azure, Oracle Cloud). There isn't a single copy of the data, as all the actors can become full nodes in the Blockchain, storing copies of it and contributing to maintaining the data integrity by using consensus algorithms. But Blockchain doesn't offer all the CRUD operations as it's a cryptographically secured immutable ledger, so it only supports create and read. Blocks can't be deleted nor updated as it would contravene Blockchain's purpose. If one node attempts to change data in a block, that will change the hash of the data from that and subsequent blocks leading to the change being rejected by the chain. Enriched with smart-contracts and a smartcontracts language (e.g., Ethereum type), the Blockchain can help support all the main processes of LFM: flexibility contracting and bidding, activation of the procured flexibility and settlement. The publish-subscribe pattern where subscribers and publishers are decoupled is 
linked to the actual implementation of the Blockchain. This allows messages to be published in a peer system on channels in order to be read by subscribers so the nodes can work in parallel without almost any coordination. The messages contain the serialized chain and are usually broadcasted when new blocks are added.

But a Blockchain can't support all the necessary processes within the LFM. Even if smartcontracts are used as part of the Blockchain (for example, to streamline payments), other solutions are needed for billing, payments outside of the Blockchain, or integrating with other parties and systems. Off-chain storage might also be necessary to comply with GDPR rules, especially with the right to be forgotten [16] and store sensitive information that you might be asked to delete. Transactional databases are very good at guaranteeing the ACID properties and following business rules. Even though NoSQL databases underpinned by the CAP theorem and focused on scale and availability started as non-relational, non-transactional, and not implementing SQL, recently, things began to change, especially for the latter two. Different NoSQL databases (DynamoDB [17], MongoDB [18]), or data warehouses (Hive [19]) began offering ACID transactions, which at first considered old-school and unnecessary. Even though this support started with limitations (single key, single shard, single region), some products dropped most of these limitations, e.g., YugabyteDB [20], which offers distributed ACID Transactions.

In Blockchain, local or sometimes global pruning is sometimes necessary in order to keep the chain at a reasonable size. Even though the process is sometimes a matter of controversy as it can lead to a loss of trust since someone can't longer verify the whole chain, it is documented in the original Nakamoto Satoshi [21] paper as a way of reclaiming disk space. In order to preserve the block's hash, transactions are hashed in a Merkle Tree with the root included in the block's hash and the interior nodes' hashes discarded. Especially in Enterprise Blockchains, there is a need to archive the whole data securely. A solution for this can be object-storage services such as S3, and its different storage classes (e.g., S3 Glacier).

\section{Conclusions}

As LFM is an important option to overcome grid, forecast and balancing issues, the setup of these markets is investigated from the ICT point of view. In the first section, a general overview regarding LFM is presented. Several researches that embrace the LFM solutions for increasing the flexibility of the power systems are mentioned in section 2. Also, in this section the LFM concept is described. In the third section, we discussed the main requirements of an LFM from an informational point-of-view, and we proposed a logical architecture to support it, explaining each of its components.

\section{Acknowledgment}

This work was supported by a grant of the Romanian National Authority for Scientific Research and Innovation, CCCDI - UEFISCDI, project title "Multi-layer aggregator solutions to facilitate optimum demand response and grid flexibility", contract number 71/2018, code: COFUND-ERANET-SMARTGRIDPLUS-SMART-MLA-1, within PNCDI III

\section{References}

[1] X. Jin, Q. Wu, and H. Jia, "Local flexibility markets: Literature review on concepts, models and clearing methods," Applied Energy. 2020, doi: 10.1016/j.apenergy.2019.114387.

[2] P. Olivella-Rosell et al., "Local flexibility market design for aggregators providing multiple flexibility services at distribution network level," Energies, 2018, doi: 10.3390/en11040822. 
[3] Y. Du and F. Li, "A Hierarchical Real-Time Balancing Market Considering MultiMicrogrids with Distributed Sustainable Resources," IEEE Trans. Sustain. Energy, 2020, doi: 10.1109/TSTE.2018.2884223.

[4] R. Poudineh and L. Boscan, "Flexibility enabling contracts in electricity markets," in 38 th IAEE International Conference, 2015.

[5] S. Ø. Ottesen, A. Tomasgard, and S. E. Fleten, "Prosumer bidding and scheduling in electricity markets," Energy, 2016, doi: 10.1016/j.energy.2015.11.047.

[6] M. Di Somma, G. Graditi, and P. Siano, "Optimal Bidding Strategy for a DER Aggregator in the Day-Ahead Market in the Presence of Demand Flexibility," IEEE Trans. Ind. Electron., 2019, doi: 10.1109/TIE.2018.2829677.

[7] P. Olivella-Rosell et al., "Optimization problem for meeting distribution system operator requests in local flexibility markets with distributed energy resources," in Applied Energy, 2018, doi: 10.1016/j.apenergy.2017.08.136.

[8] A. Ramos, C. De Jonghe, V. Gómez, and R. Belmans, "Realizing the smart grid's potential: Defining local markets for flexibility," Util. Policy, 2016, doi: 10.1016/j.jup.2016.03.006.

[9] A. Esmat, J. Usaola, and M. Á. Moreno, "A decentralized local flexibility market considering the uncertainty of demand," Energies, 2018, doi: 10.3390/en11082078.

[10] S. S. Torbaghan, N. Blaauwbroek, P. Nguyen, and M. Gibescu, "Local market framework for exploiting flexibility from the end users," in International Conference on the European Energy Market, EEM, 2016, doi: 10.1109/EEM.2016.7521304.

[11] C. Zhang et al., "A flex-market design for flexibility services through DERs," in 2013 4th IEEE/PES Innovative Smart Grid Technologies Europe, ISGT Europe 2013, 2013, doi: 10.1109/ISGTEurope.2013.6695286.

[12] S. Minniti, N. Haque, P. Nguyen, and G. Pemen, "Local markets for flexibility trading: Key stages and enablers," Energies. 2018, doi: 10.3390/en11113074.

[13] J. M. Zepter, A. Lüth, P. Crespo del Granado, and R. Egging, "Prosumer integration in wholesale electricity markets: Synergies of peer-to-peer trade and residential storage," Energy Build., 2019, doi: 10.1016/j.enbuild.2018.12.003.

[14] S. S. Torbaghan et al., "A market-based framework for demand side flexibility scheduling and dispatching," Sustainable Energy, Grids and Networks. 2018, doi: 10.1016/j.segan.2018.03.003.

[15] C. Zhang, J. Wu, Y. Zhou, M. Cheng, and C. Long, "Peer-to-Peer energy trading in a Microgrid," Appl. Energy, 2018, doi: 10.1016/j.apenergy.2018.03.010.

[16] "GDPR." [Online]. Available: https://gdpr.report/news/2018/08/06/is-gdpr-animmovable-block-to-blockchain/. [Accessed: 12-Apr-2020].

[17] "DynamoDB." [Online]. Available: https://aws.amazon.com/dynamodb/features/. [Accessed: 12-Apr-2020].

[18] "MongoDB." [Online]. Available: https://docs.mongodb.com/manual/core/transactions/. [Accessed: 12-Apr-2020].

[19] "Hive ACID." [Online]. Available: https://docs.cloudera.com/HDPDocuments/HDP3/HDP-3.1.5/using-

hiveq1/content/hive_3_internals.html. [Accessed: 12-Apr-2020].

[20] "YugabyteDB." [Online]. Available: https://github.com/yugabyte/yugabyte-db. [Accessed: 12-Apr-2020].

[21] S. Nakamoto, "Bitcoin: A Peer-to-Peer Electronic Cash System," Www.Bitcoin.Org, 2008, doi: 10.1007/s10838-008-9062-0. 